\title{
INCORPORATING PRECLINICAL AND CLINICAL KNOWLEDGE AND EXPERIENCE TO EVALUATE DRUG DEVELOPMENT PROJECTS USING THE ANALYTIC HIERARCHY PROCESS
}

\author{
A. Lawrence Gould \\ Merck Research Laboratories \\ Upper Gwynedd, PA 19454 \\ E-mail: goulda@merck.com \\ Rajesh Krishna \\ Merck Research Laboratories \\ Rahway, NJ 07065 \\ E-mail: rajesh_krishna@merck.com \\ Anis Khan \\ Medimmune, Inc. \\ Gaithersburg, MD 20878 \\ aniskhan02@yahoo.com \\ Jeffrey Saltzman \\ AstraZeneca R\&D \\ Waltham, MA 02451 \\ jeffsaltzman@gmail.com
}

\begin{abstract}
Resource limitations require choosing which candidates to progress at any stage of development to pursue, delay, or terminate. The choices must balance many dimensions such as efficacy, safety, pharmacology, market urgency, etc. The challenge is to integrate the knowledge and experience of the product development team with the findings from completed experiments and trials to inform clear, objective, and consistent development decisions. We describe, and illustrate with application to an actual development project, an approach for quantitatively assessing alternative candidates at any stage of development. The method is based on mathematical combination of sets of pairwise comparisons, which are much simpler to carry out than multi-item ranking or weighting. The dimensions of the decisions, the rankings of the possible outcomes for each dimension, and the rules for combining the components to inform a decision are determined by the project team with management concurrence before obtaining the determinative data. All of the stakeholders participate in the decision process, to assure optimum relevance to clinically oriented project teams and science-oriented management committees. Laying out the rules before a candidate reaches the point of a decision at a key point of the development process maximizes the opportunity for making the actual decision objective and transparent, and facilitates exploration of the sensitivity of the recommended decision to various assumptions. The process can be applied at any stage of clinical development. Its statistical properties can be evaluated using standard statistical decision analysis methods.
\end{abstract}


Keywords: project development decision, statistical properties,

\section{Introduction}

Product development teams and decision makers need to decide how best to allocate limited resources among sets of candidates at various stages of the drug development process using imperfect and uncertain information on multiple criteria such as safety, potential efficacy, strength of pharmacological activity, formulation feasibility, drug-drug interaction potential or changes in pharmacokinetics, commercial viability, and regulatory approvability. Conventional intuitive or subjective ranking of alternatives on multiple correlated attributes after the data have been obtained is subject to emotion, lacks transparency, does not account for uncertainty of the available information, is difficult to reproduce, and generally provides no way to assess the influence of any attribute. Especially in the current environment of limited resources for drug development, there is a clear need for an objective, transparent, and reproducible process for assessing the likelihood of success of potential candidates at every stage of development that is less likely to be driven by subjective reactions to observed data.

\section{Literature Review}

A recent survey showed that the strongest single predictor of success in drug development was a high termination rate early in development, reflecting a willingness to face hard decisions about project termination (Ringel, 2013). Various applications of decision science concepts to pharmaceutical product development have been described previously. Pallay and Berry (1999) based product development on anticipated revenue and used Bayesian models for joint efficacy and safety findings to predict future Phase 3 outcomes from Phase 2 trial findings. Burman and Senn (2003) used option values in decision models for stopping drug development, and considered the number and timing of evaluations of continuation or termination. Zipfel (2003) modeled the consequences of applying, cost-spending and net present value as stochastic processes using time-toevent models. Julious and Swank (2005) described an option-based approach to clinical development plans for individual projects. Chen and Beckman (2009) used benefit - cost ratios to support go/no go decisions for Phase 2 to Phase 3 transition and Phase 3 futility analyses in the context of oncology trials. None of these articles explained how different kinds of information from different sources reflecting different perspectives and values could be combined directly to quantify the degree of support that the accumulated information provided for specific development decisions.

\section{Hypotheses/Objectives}

The purpose of this study was to describe how the principles of the AHP approach can be used to combine clinical knowledge and experience in an objective, transparent, and reproducible way to guide decision makers, primarily at the early stages of clinical development. In addition, we sought to demonstrate how the decision process could be embedded in the context of a conventional statistical decision problem so that the statistical properties of the approach could be determined explicitly. This is important for understanding the sensitivity of the decision recommendations to the initial assumptions about the processes generating the observed data. 


\section{Research Design/Methodology}

We first identify criteria on which decisions could be based (e.g., efficacy, safety, dosage convenience, market urgency, etc.) Pairwise preferences to the criteria are determined for the criteria, leading to a set of criterion weights. We next identify a set of potential decisions that might be taken. Outcomes that could occur for each criterion are identified and, for the set of outcomes corresponding to each criterion, weights based on preference scores for the various decisions are obtained. Averaging the decision weight vectors with respect to the criterion preference weights leads to overall weight vectors for the decisions that are determined by the observed data and, subsequently, to a single weight vector for the decisions. Assumptions about the processes generating the observed outcomes define the distributions from which outcome values would be generated and these generate distributions of weight vectors corresponding to the initial assumptions that characterize the statistical properties of the approach. Given a weight vector, a choice among the decisions is made as a function of the values of the weight vector elements. There is a loss or gain attached to each such decision that depends on the initially assumed parameter values, so that one can evaluate the statistical risk associated with any set of criteria defining the process that produces the decision weight vector.

\section{Data/Model Analysis}

The details of the calculations are too lengthy to describe adequately here. They are illustrated by a consideration of how they might have been applied in the context of the actual development of a successful antidiabetic drug.

Example: End of Phase 1 evaluation of a promising antidiabetic drug

\begin{tabular}{lccccccc}
$\begin{array}{c}\text { Decision } \\
\text { criteria }\end{array}$ & $\begin{array}{c}\text { HbA1c } \\
\text { Reduc }\end{array}$ & $\begin{array}{c}\text { No hypo- } \\
\text { glycemia }\end{array}$ & $\begin{array}{c}\text { BID/QD } \\
\text { dosage }\end{array}$ & $\begin{array}{c}\text { Nowt } \\
\text { gain }\end{array}$ & $\begin{array}{c}\text { No drug-drug } \\
\text { interaction }\end{array}$ & $\begin{array}{c}\text { Can co- } \\
\text { admin. }\end{array}$ & $\begin{array}{c}\text { Market } \\
\text { Urgency }\end{array}$ \\
\hline $\begin{array}{l}\text { Typical } \\
\text { Priority Wts }\end{array}$ & 0.222 & 0.222 & 0.222 & 0.11 & 0.098 & 0.085 & 0.041 \\
& & & & & & &
\end{tabular}

Decision

\begin{tabular}{lccccc} 
& $\begin{array}{c}\text { Efficacy } \\
\text { Outcome }\end{array}$ & Phase 2b & Phase 2a & Delay & Terminate \\
\cline { 2 - 6 } $\begin{array}{l}\text { Efficacy- } \\
\text { based }\end{array}$ & No Red. & 0.05 & 0.12 & 0.26 & 0.57 \\
$\begin{array}{c}\text { Decision } \\
\text { Weights }\end{array}$ & $>20 \%$ Red. & 0.53 & 0.31 & 0.13 & 0.03
\end{tabular}

\begin{tabular}{lccccc} 
& \multicolumn{5}{c}{ Decision } \\
$\begin{array}{l}\text { Scenario- } \\
\text { based }\end{array}$ & Nenario & Phase 2b & Phase 2a & Delay & Terminate \\
\cline { 2 - 6 } $\begin{array}{l}\text { Decision } \\
\text { Weights }\end{array}$ & 0.09 & 0.18 & 0.3 & 0.43 \\
& $\begin{array}{c}\text { Mixed, } \\
\text { Somewhat }+ \\
\text { Highly }+\end{array}$ & 0.38 & 0.42 & 0.21 & 0.1 \\
& 0.55 & 0.27 & 0.13 & 0.04
\end{tabular}

The actual outcomes were consistent with a highly positive scenario, so that the weight for proceeding with aggressive development was 0.55 and the weight for proceeding with development whether aggressively or not was 0.82 , which strongly supports proceeding.

International Symposium of the Analytic Hierarchy

Process
Washington, D. C. June 29 - July 2, 2014 


\section{Limitations}

The major limitation of this approach arises from the assumption that the decision alternatives and the decision criteria are complete and well defined. Obtaining these is unlikely to be a trivial exercise in practice. It may be easier to achieve agreement regarding pairwise preference scores by asking individuals to provide ranges of preference scores rather than specific values, and simulation can be used to arrive at consensus scores and weights that have probabilistic interpretations.

\section{Conclusions}

Our major objective was to describe how AHP methods could be integrated with statistical decision methodology to inform project development decisions. We used a simple example to illustrate how results from trials could support alternative decisions. The results of this evaluation suggest that the AHP approach can be employed in the drug development process, certainly at the earliest stages when rigorous decisions need to be made. Regardless of how decisions are evaluated, a rational, transparent decision process requires deciding how accumulated information from each of the decision dimensions will guide the process. These decisions currently appear often to be done intuitively without explicit statement of assumptions or consideration of consequences. Quantifying the process using AHP should improve the transparency of the decision process and allow the consequences of different assumptions to be evaluated objectively.

\section{Key References (not including references to AHP literature)}

Pallay, A., Berry, S.M. (1999) A decision analysis for an end of Phase II go/stop decision. Drug Information Journal, 33, 821-833.

Burman, C.F., Senn, S. (2003) Examples of option values in drug development. Pharmaceutical Statistics, 2, 113-125.

Zipfel, A. (2003) Modeling the probability-cost-profitability architecture of portfolio management in the pharmaceutical industry. Drug Information Journal, 37, 185-205.

Julious, S.A., Swank, D.J. (2005) Moving statistics beyond the individual clinical trial: Applying decision science to optimize a clinical development plan. Pharmaceutical Statistics, 4, 37-46.

Chen, C., Beckman, R.A. (2009) Optimal cost-effective go-no go decisions in late-stage oncology drug development. Statistics in Biopharmaceutical Research, 1, 159-169.

Ringel, M., Tollman, P., Hersch, G., Schulze, U. (2013) Does size matter in R\&D productivity? If not, what does? Nature Reviews/Drug Discovery, 12, 901902. 